\title{
Cardiac Inotropes Offer Protection of Renal Function in Patients with Kidney Transplantation
}

\author{
Ning Guo ${ }^{a}$ Zehua Wang ${ }^{d}$ Laurine M. Bow ${ }^{\text {b, c }}$ Xianquan Cui ${ }^{a}$ \\ Luwei Zhang $^{a}$ Wanhua Xian ${ }^{a}$ Huaibin Sun $^{a}$ Jun Tian ${ }^{a}$ \\ aDepartment of Surgery and Transplantation, Qilu Hospital, Shandong University,

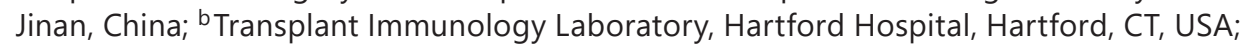 \\ 'Department of Transplantation Surgery, Yale University School of Medicine, New Haven, \\ CT, USA; ${ }^{d}$ Department of Urology Surgery, Qilu Hospital, Shandong University, Jinan, China
}

\section{Keywords}

Kidney transplantation · Cardiac inotropes · Kidney function

\begin{abstract}
Introduction: Impaired cardiac function is one of the most concomitant symptoms in patients with kidney failure after long-term dialysis. In addition, the preservation of adequate perfusion pressure to the graft plays a significant role in the intraoperative management during kidney transplantation, but the use of positive inotropic drugs in kidney transplant patients has been studied less. We investigated the protective effects of renal function by means of cardiac inotropes in kidney transplant patients. Methods: Eighty-nine patients that received kidney transplantation between April 2014 and December 2016 at Qilu Hospital were included and randomly divided into the treatment group receiving levosimendan and a control group. All kidney recipients received $\mathrm{ABO}$-compatible donors. A poor outcome was defined as one of the following: delayed graft function, graft hemorrhage, or nephrectomy. Results: The treatment group had a better composite outcome and the level of neutrophil gelatinaseassociated lipocalin was also lower than in the control group. Conclusion: Inotropic drugs may play a protective role in renal function in kidney transplantation.
\end{abstract}

(C) 2020 The Author(s)

Published by S. Karger AG, Basel

Ning Guo and Zehua Wang contributed equally to this work and should be considered co-first authors.

Jun Tian

Department of Surgery, Transplantation, Qilu Hospital, Shandong University

WenHuaXi Road \#107

Jinan 250012, Shandong (China)

E-Mail juntian63@ sina.com 


\section{Kidney \\ Blood Pressure \\ Research}

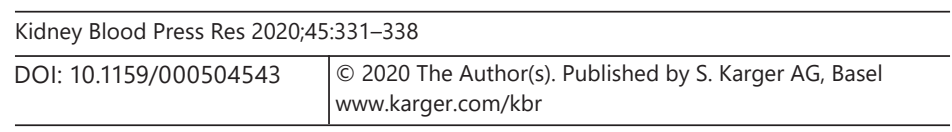

Guo et al.: Cardiac Inotropes Offer Protection of Renal Function in Patients with Kidney Transplantation

\section{Background}

The interaction between the heart and kidneys maintains the stability of blood volume, blood vessel tension, and hemodynamics. Existing studies have not yet explored the relationship between heart function and renal failure. However, the concept of cardiorenal syndrome is known, and therefore cardiac and renal function are thought to be closely related [1]. This relationship is particularly important in long-term dialysis patients because most patients with uremia have impaired heart function, and successful kidney transplantation is crucial for the prognosis of uremic patients. After transplantation, good cardiac function and blood flow perfusion are two of the most important factors for the restoration of renal function. Therefore, the use of inotropes to maintain kidney perfusion after kidney transplantation is an issue worth exploring.

Because the causes of heart failure vary, the effects of inotropic cardiac drug therapy in patients are not the same. As is known, beneficial inotropic drugs include cardiac glycosides and nonglucosides (including phosphodiesterase inhibitors, calcium sensitizers, $\beta$ agonists, etc.). An early study demonstrated that dobutamine and milrinone can increase heart function and renal blood flow and decrease the incidence of acute renal injury after heart surgery, suggesting that these drugs protect the kidneys [2]. However, the results of other studies show that inotropic drugs show no direct improvement of renal function and prognosis in patients with chronic heart failure $[3,4]$. These results suggest to us that the protective effect of cardiac drugs on heart failure has not been confirmed. At present, new types of inotropic cardiodrugs are under development. As a new class of calcium sensitizer inotropic drug, levosimendan can improve cardiac function in patients with cardiac insufficiency, but only a few studies have evaluated its effectiveness in improving kidney function. This study was aimed at evaluating the effectiveness of cardiac inotropes, specifically levosimendan, by measuring the protective effects of this drug on renal function in kidney transplant patients.

\section{Material and Methods}

\section{Patients and Inclusion/Exclusion Criteria}

Eighty-nine patients who underwent kidney transplantation between April 2014 and December 2016 at the Qilu Hospital were included in this study. Inclusion criteria were: (1) level 2 cardiac insufficiency according to the New York Heart Association (NYHA) standard, (2) transplant from an ABO-compatible donor, (3) a negative panel reactive antibody (PRA) test and cross-match at the time of transplantation, and (4) an age at transplantion of 4-70 years. Exclusion criteria were: (1) mental disorders and cognitive dysfunction, (2) allergic diseases and/or allergies, and (3) severe aphasia, agnosia, or apraxia. All patients were followed up for outcomes until June 2017. Clinical data were collected from enrollment and throughout follow-up.

The 2 main clinical outcomes compared in this study were survival and composite clinical outcome. A poor composite outcome was defined as any 1 of the following: death, a blood creatinine level $>500 \mu \mathrm{mol} / \mathrm{L}$, a return to dialysis, or a rejection episode. A good composite outcome was when patients did not exhibit any of these criteria. A diagnosis of acute rejection was based upon biopsy results.

\section{Treatment}

Patients were randomly assigned to 2 groups. The treatment group underwent a course of standard therapy after kidney transplantation including administration of the inotrope levosimendan. The control group underwent the standard therapy but did not receive the 


\section{Kidney \\ Blood Pressure \\ Research}

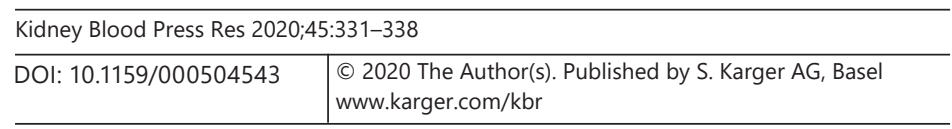

Guo et al.: Cardiac Inotropes Offer Protection of Renal Function in Patients with Kidney Transplantation

inotrope. All patients received standard triple immunosuppression therapy, i.e., calcineurin inhibitors, mycophenolatemofetil, and prednisone. Desensitization treatment strategies were not deployed for any patients.

Levosimendan was initiated at a dose of 6-12 $\mu \mathrm{g} / \mathrm{kg}$ intravenously, and then resumed at $0.1 \mu \mathrm{g} / \mathrm{kg} / \mathrm{min}$ for $1 \mathrm{~h}$. After that, the dosage was adjusted to $0.2 \mu \mathrm{g} / \mathrm{kg} / \mathrm{min}$ and maintained for $24 \mathrm{~h}$. All treatment was carried out under electrocardiographic monitoring. If any of the following occurred, the treatment was suspended: (1) a heart rate $>130$ beats per minute (bpm), that increased by $>15 \mathrm{bpm}$ or lasted for $>10 \mathrm{~min}$; (2) symptomatic hypotension or a systolic blood pressure of < $75 \mathrm{~mm} \mathrm{Hg}$; (3) malignant arrhythmia; (4) adverse events that the investigators considered significant enough to discontinue treatment.

\section{Concomitant Medication}

Patients were permitted to accept an increase in drug dose, diuretics, and any other basic drug therapy.

\section{Observation Index}

Clinical Signs and Symptoms. This involved a serious change in vital signs or the occurrence of complications (e.g., delayed graft function, graft hemorrhage, rupture or nephrectomy, and death). The monitoring period was 1 year after kidney transplantation.

Renal Function-Related Index. This involved urine volume and serum creatinine (Scr) level after $24 \mathrm{~h}$. These indices were monitored in the hospital lab each day for 7 days, and then at 2 weeks, 1 month, and 3 months.

Renal Injury-Related Index. This involved the expression of neutrophil gelatinase-associated lipocalin (NGAL). The concentration of NGAL was measured using a human ELISA kit according to the manufacturer's instructions. The kit was bought from Cusabio (cat. No. CSBE09408h). The absorbance (OD value) of each hole was measured at a 450-nm wavelength, and the concentration measured according to the OD value of serum samples with a standard curve.

\section{Statistics}

Differences in quantitative parameters were assessed using the $t$ test for data that were normally distributed or the nonparametric test for data that were not normally distributed. Differences in qualitative data were compared using the $\chi^{2}$ test and Fisher's exact test. KaplanMeier curves and log rank tests were performed to compare survival and composite outcomes between groups. $p<0.05$ was considered significant. Statistical analysis was performed with SPSS v15.0 (SPSS Inc., Chicago, IL, USA).

\section{Results}

Of 89 kidney transplant recipients, 45 received standard therapy with levosimendan after kidney transplantation (treatment group), and the other 44 received standard therapy without inotropes (control group).

The clinical baseline data of these patients appear in Table 1. There were no significant differences between groups in age, sex, or blood transfusion frequency, donor type, pretransplant creatinine level, or HLA mismatch. There was also no significant difference in survival between the treatment and control groups (Table 2). One patient died on day 7 after transplantation due to a lung infarction and the other died 6 months later in a car accident. There was no significant difference in the recovery of renal function after kidney transplantation as indicated by the level of creatinine and the volume of urine after $24 \mathrm{~h}$ (Fig. 1, 2). 
Table 1. Demographic and clinical data

\begin{tabular}{llll}
\hline & $\begin{array}{l}\text { Treatment group } \\
(n=45)\end{array}$ & $\begin{array}{l}\text { Control group } \\
(n=44)\end{array}$ & $p$ value \\
\hline Age, years & $43.62 \pm 11.22$ & $48.55 \pm 14.42$ & 0.621 \\
Gender, male/female & $28 / 17$ & $26 / 18$ & 0.731 \\
Transfusions (no/yes) & $41 / 4$ & $38 / 6$ & 0.892 \\
Race (Han/other) & $44 / 1$ & $42 / 2$ & 0.773 \\
Pretransplant creatinine, $\mu \mathrm{mol} / \mathrm{L}$ & $876.2 \pm 153.07$ & $989.34 \pm 133.55$ & 0.804 \\
Donor type, live/DCD & $22 / 23$ & $19 / 25$ & 0.793 \\
\hline
\end{tabular}

Values express $n$ or mean \pm SD. DCD, donation after cardiac death.

Table 2. Survival rate results

\begin{tabular}{llll}
\hline & Deaths, $n$ & $\begin{array}{l}\text { Patients who } \\
\text { survived, } n\end{array}$ & \\
\hline $\begin{array}{l}\text { Treatment group } \\
\text { Control group }\end{array}$ & 0 & 45 & $p>0.05$ \\
$\chi^{\text {a }}$ & 42 & $\chi^{2}=0.53$ \\
\hline
\end{tabular}

a One patient died on day 7 after transplantation due to a lung infarction and the other 6 months later in a car accident.

Table 3. Composite results

\begin{tabular}{llll}
\hline & $\begin{array}{l}\text { Good composite } \\
\text { outcome }\end{array}$ & $\begin{array}{l}\text { Poor composite } \\
\text { outcome }\end{array}$ \\
\hline $\begin{array}{l}\text { Treatment group } \\
\text { Control group }\end{array}$ & 42 & 3 & $p<0.05$ \\
\hline
\end{tabular}

However, the level of NGAL was lower in the treatment group during the first week after transplantation (Fig. 3). The treatment group also had a better composite outcome than the control group (Table 3).

\section{Discussion}

In this study, due to the polyuria stage during the first few days (usually starting on days 1-3 and reaching a peak on day 4 or 5), urine volume in both groups increased on days 4 and 5 and then gradually decreased. As for the success of the kidney transplantation, the creatinine values in both groups were normal after 1 week. Although there was no statistical difference in creatinine values, urine volume, or survival rate between the 2 groups, the treatment group had a better composite outcome than the control group, suggesting that inotropic agents can affect the long-term prognosis after kidney transplantation. The index of renal injury, the NGAL level, was higher in the control group than in the treatment group, also indicating that inotropic agents play a role in kidney protection during the transplantation process.

As previously known, inotropic drugs can activate the $\mathrm{K}^{+}$channel of vascular smoothmuscle cells, reduce the time of action potential, and prolong the occurrence of hyperpolar- 
Kidney

Blood Pressure

Research

Fig. 1. Creatinine levels after renal transplantation in the treatment and control groups.

Fig. 2. Urine volumes after renal transplantation in the treatment and control groups.

Fig. 3. The expression of neutrophil gelatinase-associated lipocalin (NGAL) after renal transplantation in the treatment and control groups. There were statistical differences only on days 1-7.

\begin{tabular}{l|l}
\hline Kidney Blood Press Res 2020;45:331-338 \\
\hline DOI: 10.1159/000504543 & $\begin{array}{l}\text { @ 2020 The Author(s). Published by S. Karger AG, Basel } \\
\text { www.karger.com/kbr }\end{array}$ \\
\hline
\end{tabular}

Guo et al.: Cardiac Inotropes Offer Protection of Renal Function in Patients with Kidney Transplantation
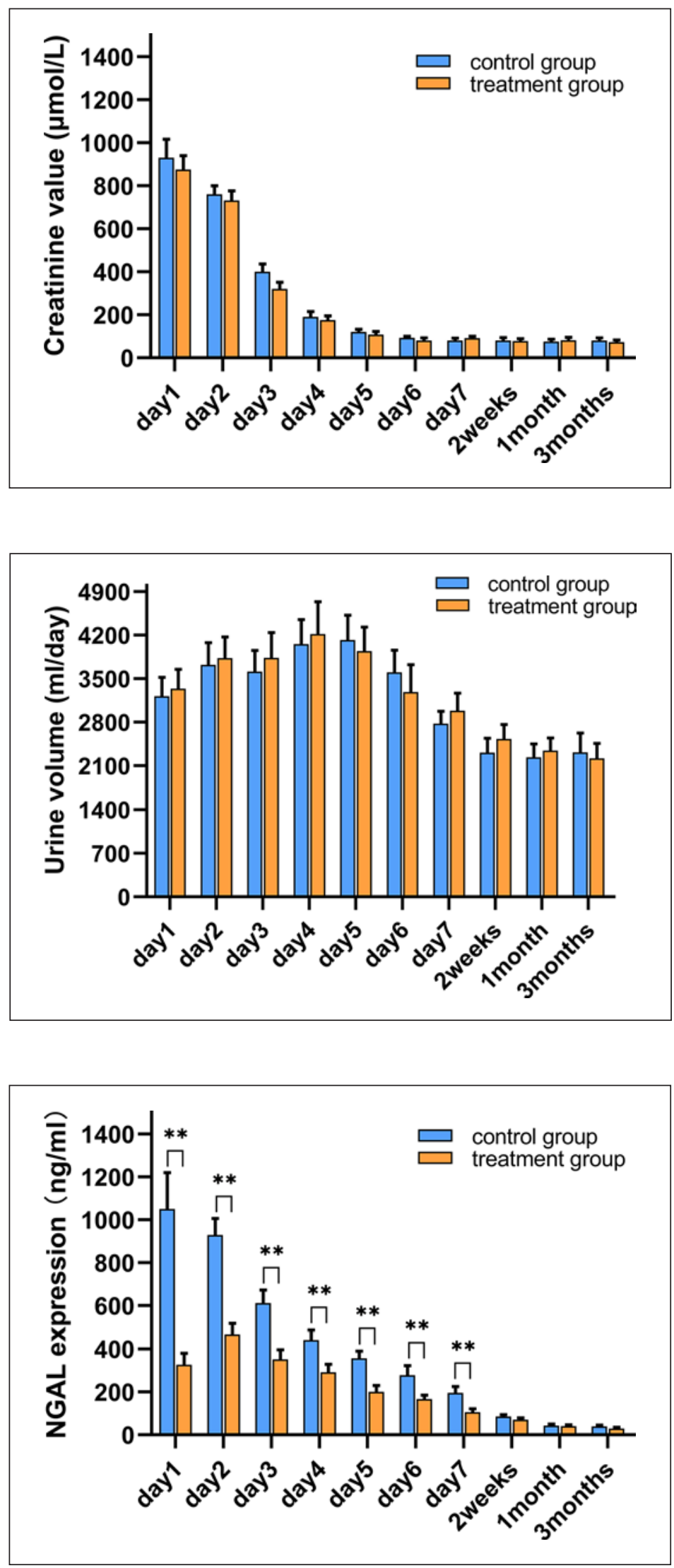


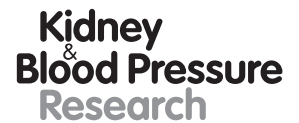

Research

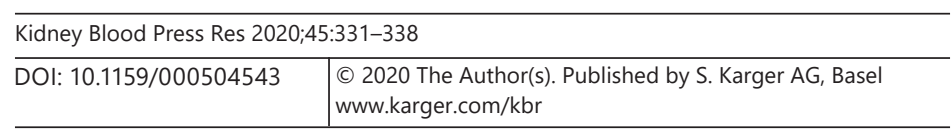

Guo et al.: Cardiac Inotropes Offer Protection of Renal Function in Patients with Kidney Transplantation

ization, which lead to an extension of $\mathrm{Ca}^{2+}$ channel and a decrease in calcium influx [5]. Furthermore, these drugs can activate the $\mathrm{Na}^{+}-\mathrm{Ca}^{2+}$ protein complex, which can increase calcium efflux and reduce intracellular $\mathrm{Ca}^{2+}$ concentration. This can promote the relaxation of arterial smooth-muscle cells and the expansion of small arteries, which upregulate the perfusion of blood into tissues and organs [6]. Yilmaz et al. [7] showed that levosimendan improved renal function in 88 patients with acute decompensating heart failure, and pointed out that the improvement in glomerular filtration rate (GFR) induced by levosimendan lasted for $72 \mathrm{~h}$. In addition, levosimendan can cause the relaxation of arteries and veins [8], including the central and peripheral blood vessels. A previous study showed that levosimendan played a decisive role in controlling central venous pressure, an important independent predictor of GFR in patients with heart failure [9]. As a result, the dilatation of renal blood vessels increases blood perfusion from the renal arteries and reduces the risk of renal ischemia, eventually improving renal function [10,11]. Our assumption is that inotropic drugs may have a protective effect on the kidneys after kidney transplantation. This would explain our observation that the treatment group had a better composite outcome than the control group.

In our study, levosimendan was delivered within $24 \mathrm{~h}$ by means of a single dose. this drug has a long half-life of about $80 \mathrm{~h}$. Consequently, it can be expected that a 24 -h intravenous injection will maintain the treatment effect for approximately 1 week [12]. According to previous studies, levosimendan is more effective than dobutamine for improving hemodynamic parameters in patients with congestive heart failure [10, 13]. Strong myocardial contraction and stable dynamic blood flow are expected to maintain sufficient blood perfusion to the kidneys $[14,15]$. Our study suggests that this mechanism is also effective in renal transplantation. Previous univariate analysis suggested that increased hemodynamics and augmentation of renal perfusion may be important mechanisms to explain the improvement of renal function in patients treated with levosimendan [16]. For systemic inflammatory responses caused by severe infection or other reasons, levosimendan can reduce the degree of inflammatory reactivity by decreasing the release of inflammatory cytokines, and consequently protect organ function [17-20]. In addition, because of its anti-inflammatory effects, levosimendan can help reduce the dosage of immunosuppressive agents after renal transplantation, thereby reducing renal toxicity and playing a protective role for the transplanted kidney. All of the above could explain why NGAL levels were reduced in the treatment group.

Levosimendan promotes the transformation of L-arginine to nitric oxide (NO) in endothelial cells by increasing NO synthase in the blood vessels. NO is known to expand the coronary arteries and also participates in the inflammatory reactivity of the myocardium, by reducing the aggregation of inflammatory cells, inhibiting the aggregation and adhesion of platelets, and preventing intravascular thrombosis [21]. These indirect anti-inflammatory and antithrombotic effects are very important for the long-term protection and function of the renal allograft.

In conclusion, we have shown that the long-term prognosis after renal transplantation can be improved by the use of isotropic drugs (e.g., levosimendan), suggesting a protective effect in renal injury after kidney transplantation. This mechanism may be related to an improvement in cardiac function (that causes the expansion of the renal vasculature) and the renal allograft blood supply. It must be noted that the number of patients in this study was small and the observation time was short. Further investigation will be needed to determine the long-term efficacy of inotropic therapy on kidney graft function after transplantation.

\section{Acknowledgement}

We would like to show our deep gratitude to all doctors and nurses of our department. Without their kindness, patience, and hard work, we could not have completed this paper. 


\section{Kidney \\ Blood Pressure \\ Research}

\begin{tabular}{l|l}
\hline Kidney Blood Press Res 2020;45:331-338 \\
\hline DOI: 10.1159/000504543 & $\begin{array}{l}\text { ○ 2020 The Author(s). Published by S. Karger AG, Basel } \\
\text { www.karger.com/kbr }\end{array}$ \\
\hline
\end{tabular}

Guo et al.: Cardiac Inotropes Offer Protection of Renal Function in Patients with Kidney Transplantation

\section{Statement of Ethics}

Subjects (or their parents or guardians) gave their written informed consent. The study protocol was approved by the Qilu Hospital committee on human research (reg. No. 2013009).

\section{Disclosure Statement}

The authors have no conflicts of interest to declare.

\section{Funding Sources}

This research was supported by a ShanDong Science and Technology development project (No. 2014GSF121017) and a transverse project (No. 26010111671305).

\section{Author Contributions}

Ning Guo and Jun Tian designed the experiment, Ning Guo and Laurine wrote the article, and data collection and statistical analysis were completed by Zehua Wang, Xianquan Cui, Luwei Zhang, Wanhua Xian, Huaibin Sun, and Jun Tian.

\section{References}

1 McAlister FA, Ezekowitz J, Tonelli M, Armstrong PW. Renal insufficiency and heart failure: prognostic and therapeutic implications from a prospective cohort study. Circulation. 2004 Mar;109(8):1004-9.

2 Kurien S, Warfield KT, Wood CM, Miller WL. Effects of standard heart failure therapy and concomitant treatment with intravenous furosemide or inotropes (dobutamine, dopamine, and/or milrinone) on renal function and mortality in patients treated with nesiritide. Am J Cardiol. 2006 Dec;98(12):1627-30.

3 Klein L, Massie BM, Leimberger JD, O'Connor CM, Piña IL, Adams KF Jr, et al.; OPTIME-CHF Investigators. Admission or changes in renal function during hospitalization for worsening heart failure predict postdischarge survival: results from the Outcomes of a Prospective Trial of Intravenous Milrinone for Exacerbations of Chronic Heart Failure (OPTIME-CHF). Circ Heart Fail. 2008 May;1(1):25-33.

4 Thackray S, Easthaugh J, Freemantle N, Cleland JG. The effectiveness and relative effectiveness of intravenous inotropic drugs acting through the adrenergic pathway in patients with heart failure-a meta-regression analysis. Eur J Heart Fail. 2002 Aug;4(4):515-29.

5 Yokoshiki H, Katsube Y, Sunagawa M, Sperelakis N. Levosimendan, a novel Ca2+ sensitizer, activates the glibenclamide-sensitive K+ channel in rat arterial myocytes. Eur J Pharmacol. 1997 Aug;333(2-3):249-59.

6 Du Toit EF, Muller CA, McCarthy J, Opie LH. Levosimendan: effects of a calcium sensitizer on function and arrhythmias and cyclic nucleotide levels during ischemia/reperfusion in the Langendorff-perfused guinea pig heart. J Pharmacol Exp Ther. 1999 Aug;290(2):505-14.

7 Yilmaz MB, Yalta K, Yontar C, Karadas F, Erdem A, Turgut 00, et al. Levosimendan improves renal function in patients with acute decompensated heart failure: comparison with dobutamine. Cardiovasc Drugs Ther. 2007 Dec;21(6):431-5.

8 Parissis JT, Farmakis D, Nieminen M. Classical inotropes and new cardiac enhancers. Heart Fail Rev. 2007 Jun; 12(2):149-56.

9 Damman K, Navis G, Voors AA, Asselbergs FW, Smilde TD, Cleland JG, et al. Worsening renal function and prognosis in heart failure: systematic review and meta-analysis. J Card Fail. 2007 Oct;13(8):599-608.

10 Maytin M, Colucci WS. Cardioprotection: a new paradigm inthe management of acute heart failure syndromes. Am J Cardiol. 2005;96(6 6A):26-31.

11 Follath F, Cleland JG, Just H, Papp JG, Scholz H, Peuhkurinen K, et al.; Steering Committee and Investigators of the Levosimendan Infusion versus Dobutamine (LIDO) Study. Efficacy and safety of intravenous levosimendan compared with dobutamine in severe low-output heart failure (the LIDO study): a randomised double-blind trial. Lancet. 2002 Jul;360(9328):196-202.

12 Dec GW. Acute decompensated heart failure: the shrinking role of inotropic therapy. J Am Coll Cardiol. 2005 Jul;46(1):65-7. 
13 Kivikko M, Lehtonen L, Colucci WS. Sustained hemodynamic effects of intravenous levosimendan. Circulation. 2003 Jan;107(1):81-6.

14 Sorsa T, Heikkinen S, Abbott MB, Abusamhadneh E, Laakso T, Tilgmann C, et al. Binding of levosimendan, a calcium sensitizer, to cardiac troponin C. J Biol Chem. 2001 Mar;276(12):9337-43.

15 Toivonen L, Viitasalo M, Sundberg S, Akkila J, Lehtonen L. Electrophysiologic effects of a calcium sensitizer inotrope levosimendan administered intravenously in patients with normal cardiac function. J Cardiovasc Pharmacol. 2000 Apr;35(4):664-9.

16 Zemljic G, Bunc M, Yazdanbakhsh AP, Vrtovec B. Levosimendan improves renal function in patients with advanced chronic heart failure awaiting cardiac transplantation. J Card Fail. 2007 Aug;13(6):417-21.

17 Follath F, Cleland JG, Just H, et al. Efficacy and safety of intravenous levosimendan compared with dubutamine in severe low output heart failure (the LIDO study): a randomized double-blind trial. Lancet. 2002;360:196202.

18 Moiseyev VS, Poder P, Andrejevs N. Safety and efficacy of a novel calcium sensitizer, levosimendan in patients with left ventricular failure due to an acute myocardial infarction. A randomized, placebo-controlled, doubleblind study (RUSSLAN). Eur Heart J. 2002;23:1422-32.

19 Cleland JG, Ghosh J, Freemantle N. Clinical trials update and cumulative meta-analyses from the American College of Cardiology: WATCH, SCD-HeFT, DINAMIT, CASINO, INSPIRE, STRATUS-US, RIO-Lipids and cardiac resynchronization therapy in heart failure. Eur J Heart Fail. 2004;6:501-8.

20 Biondi-Zoccai GG, Agostoni P, Abbate A, Testa L, Burzotta F. A simple hint to improve Robinson and Dickersin's highly sensitive PubMed search strategy for controlled clinical trials. Int J Epidemiol. 2005 Feb;34(1):224-5.

21 Grossini E, Caimmi PP, Molinari C, Teodori G, Vacca G. Hemodynamic effect of intracoronary administration of levosimendan in the anesthetized pig. J Cardiovasc Pharmacol. 2005 Sep;46(3):333-42. 